\title{
BRIDGE PAVEMENT CRACK DETECTION UNDER UNEVEN ILLUMINATION USING IMPROVED PCNN ALGORITHM
}

\author{
Shiling $\mathrm{Su}^{1^{*}}$, Tao Gong ${ }^{1,2}$ \\ ${ }^{1}$ College of Earth Sciences and Environmental Engineering, Southwest Jiaotong University \\ ${ }^{2}$ Department of Surveying Engineering, Southwest Jiaotong University, Chengdu
}

KEY WORDS: Uneven Illumination; Bridge Pavement Crack; Cross-Bilateral Filtering; SPCNN

\begin{abstract}
:
For bridge pavement cracks under uneven illumination, the existing image segmentation algorithm does not remove this effect, and the segmentation effect is affected. In this paper, the image preprocessing consists of two parts: the process of removing uneven illumination and image noise, and the traditional bilateral filtering is improved based on the stationary wavelet algorithm (Cross-bilateral filtering). In the image segmentation part, the traditional PCNN (Pulse Coupled Neural Network) model parameters and the number of iterations are difficult to determine reasonably, and the use of a certain complexity makes it difficult to automate. This paper combined the synaptic integration characteristics of neurons, image gray and spatial features, to simplify PCNN model. The improved PCNN algorithm (SPCNN) based on the gray threshold of Markov network directly completes the segmentation without the need to manually set parameters and determine the optimal number of iterations. Through the analysis of the experimental results, the following three conclusions were drawn. (1) Compared with the histogram equalization, the enhancement algorithm of this paper removed the influence of illumination well and had advantages for the subsequent segmentation processing. (2) The cross-bilateral filtering algorithm could improve the image signal-to-noise ratio from 18.855 to 32.037 , which was better than the original bilateral filtering algorithm. (3) The average segmentation accuracy rate of segmentation of SPCNN algorithm was more that $90 \%$. Compared with the traditional PCNN method, this method is better in subjective visual effects and objective segmentation performance, less time consuming.
\end{abstract}

\section{INTRODUCTION}

The total number of highway bridges in China exceeds 830,000. The occurrence of bridge collapse accidents will not only cause huge economic losses, but also hurt many innocent people. Therefore, our country pays more attention to road and bridge maintenance work. Relevant historical data showed that bridge safety accidents caused by cracks account for about $90 \%$ of the total disasters of bridges. And pavement crack detection has become a core part of maintenance work. The existing bridge crack detection methods can be roughly divided into three methods: manual detection, large-scale inspection vehicle detection and non-destructive testing technology. The first two detection methods are completely dependent on the operator, affecting traffic during operation, have safety hazards and low efficiency. With the development of computer vision

* Corresponding author: Shiling Su,

Email: 1378495266@qq.com technology, non-destructive testing has gradually become the mainstream crack detection technology, which can overcome the shortcomings of manual methods.

The detection process of bridge crack images is generally composed of pre-processing, description and recognition. The effect of automatic detection of crack images on highway bridges depends on the image quality and the description of the cracks in the image. In the case of uniform pavement materials, clean road surface and good image collection environment, the acquired image texture is clear and the crack visual significance is high(Zhang et al.,2016). For such road crack images, the methods of classical gray threshold segmentation(He et al.,2012), morphology(Chambon et al.,2011;Lee et al.,2013), and machine learning(Oliveira et al.,2013) could be used to detect cracks automatically and achieve good results. However, affected by weather, light, paving materials and road conditions in the actual situation, the obtained crack images may have some phenomena that uneven illumination, insufficient exposure or overexposure, blurring, and there are still many 
noises. Yusuke et al.(2006) used image smoothing to remove the effects of uneven illumination and noise, then used threshold segmentation to extract crack information. The algorithm could successfully extract the crack information on a part of the concrete images, but the method of fixed threshold was poor in flexibility and the application surface was narrow. Nishikawa et al.(2012) designed a special image filter to suppress noise by genetic programming, detected a large number of crack regions by image filter method for the first time, and then reused it in the local area around the crack target to eliminate residual noise. However, this method was easy to break the crack and affect the extraction of the crack target contour. Liu et al.(2008) proposed an automatic crack detection method for complex pavement images based on segmentation expansion. By analyzing the relationship between connected regions, the correlated segments formed cracks by joining, and crack direction features could be better used for crack identification. Wang et al.(2015) added the crack location information to the clustering algorithm, and combined the pixel values of the crack to comprehensively judge the crack region, the problem that crack information difficult to identify due to uneven illumination during image acquisition was overcame. But the algorithm only could deal with a single crack, which has limitations. Li et al.(2010) proposed a detection algorithm based on minimum path selection for low pavement crack contrast and poor continuity, which better solved the problem of detecting and extracting pavement cracks under low resolution, however it did not involve the extraction of cracks under complex conditions. In recent years, deep learning has begun to rise. The use of convolutional neural networks for crack detection(Zhao et al.,2017) had high recognition and classification accuracy, but it was difficult to achieve because of requiring a large number of training learning processes, samples and high cost.

On the basis of fully analyzing the characteristics of concrete bridge pavement cracks, this paper firstly preprocessed the images under uneven illumination. The pre-processing process included removing the illumination effect and using the crossbilateral filtering to smooth images. Then used the global and local gradation characteristics of the crack distribution, a simplified PCNN model based on fixed threshold determined by the Markov network (SPCNN) is proposed to perform image segmentation. The experimental results not only showed that the proposed algorithm had better detection performance for the targets in the uneven illumination bridge crack images, but also had better robustness for various changes of images.

\section{CRACK DETECTION OF UNEVEN ILLUMINATION BRIDGE PAVEMENT BY SPCNN}

\subsection{Remove Uneven Illumination}

\subsubsection{The Derivation Process}

The image can be represented by a two-dimensional luminance function $\mathrm{f}(\mathrm{x}, \mathrm{y})$ formed by reflected light on the object. Assuming that reflected light is equal to incident light multiplied by the reflection coefficient, $\mathrm{f}(\mathrm{x}, \mathrm{y})$ depends on the amount of incident light $\mathrm{i}(\mathrm{x}, \mathrm{y})$ on the scene and reflectivity $\mathrm{r}(\mathrm{x}$, y) of the object. The equation of $f(x, y)$ can be expressed as:

$$
f(x, y)=i(x, y) * r(x, y)
$$

In theory, $0<i(x, y)<+\infty, 0<r(x, y)<1$ 。 According to equation (1), $r(x, y)=f(x, y) / i(x, y)$ could be obtained. Through further analysis we found that the illumination function $\mathrm{i}(\mathrm{x}, \mathrm{y})$ changed slowly in space, and the reflection function $\mathrm{r}(\mathrm{x}, \mathrm{y})$ would abruptly change at the edge of the object. It could be considered that the low frequency part of $f(x, y)$ mainly affected by $\mathrm{i}(\mathrm{x}, \mathrm{y})$, and $\mathrm{r}(\mathrm{x}, \mathrm{y})$ was reflected in the high frequency part of $f(x, y)$. Therefore, the spatial distribution of $\mathrm{i}(\mathrm{x}, \mathrm{y})$ could be approximated by the low-frequency part of the image $f(x, y)$ (Wu et al.,2010). In this paper, wavelet analysis was performed on $\mathrm{f}(\mathrm{x}, \mathrm{y})$, and the low-frequency part was extracted as an estimate of $\mathrm{i}(\mathrm{x}, \mathrm{y})$. An image that removed the influence of illumination (ie, a reflection function) could be obtained according to the above equation. However, in practical applications, some restrictions needed to be added. In order to avoiding the value of the above formula is too large, the value of denominator $\mathrm{i}(\mathrm{x}, \mathrm{y})$ was greater than or equal to 1 , and $\mathrm{r}(\mathrm{x}, \mathrm{y})$ was relaxed from $[0,1]$ to $[0,2]$. Finally, the reflection function was linearly stretched to the $[0,255]$ to achieve image enhancement.

\subsubsection{Comparison of Results of Histogram Equalization and} Remove Uneven Illumination Algorithm

For the image of the crack on the surface of the bridge, the image is sometimes dark due to insufficient light or insufficient exposure, or the image is partially over-bright due to uneven illumination. The images in these two cases were selected to 
perform histogram equalization(HE) and the de-lighting influence algorithm. After many experiments, the remove uneven illumination(RUI) algorithm selected the "coif3" wavelet, and the decomposition layer was 5 to perform wavelet decomposition to obtain the de-illuminated image results that was best. After the dark crack image (named test1) and the uneven light image (named test2) were respectively subjected to histogram equalization and remove uneven illumination algorithm, which could be found that the histogram equalized image became brighter, but the background gray level was uneven, would affect target extraction; the image of removing uneven illumination and it's background gray were even, at the same time the target of image was effectively highlighted, it was of great help to the subsequent segmentation process. Therefore, the enhancement method of this paper is better than traditional histogram equalization, the results were shown in Figure 1:

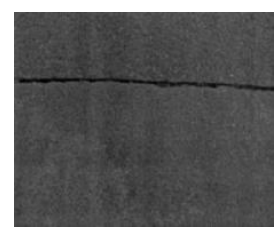

(a1) test1

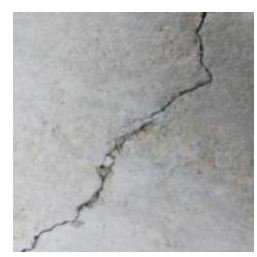

(b1) test2

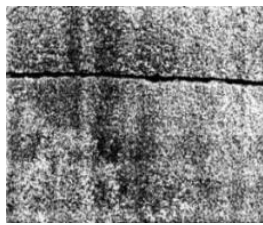

(a2) HE_test1

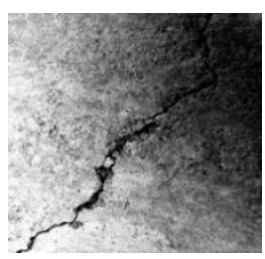

(b2) HE_test2

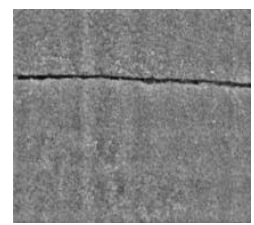

(a3) RUI_test1

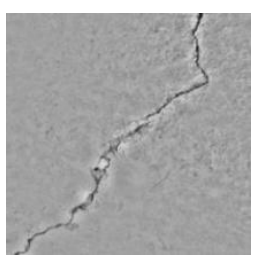

(b3) RUI_test2
Figure 1. Results of the histogram equalization and remove uneven illumination algorithm

\subsection{Cross-Bilateral Filtering (Cross-BF)}

The Gaussian filter weight uses the spatial distance measure factor: the farther the surrounding pixel is from the center point, the smaller the weight. The Yaroslavsky filter weight depends on the gray similarity: the closer the surrounding pixel points are to the center point gray value, the larger the weight. The bilateral filtering algorithm (BF) combines the spatial distance measure factor of Gaussian filtering with the grayscale measure factor of Yaroslavsky filtering, which preserves the edge details well when the image is smoothed. Bilateral filter grayscale measure factor calculates the gray difference between two independent pixels, when two adjacent pixels are polluted by noise, the stability of the grayscale measure factor calculation between the center pixel and the neighborhood pixel will be drop. Therefore, the key to improving the performance of the weighted average algorithm such as bilateral filtering is to improve the accuracy of the grayscale measurement weight. In this paper, a cross-bilateral filtering algorithm is proposed. The stationary wavelet algorithm is used as a pre-filter, the grayscale measurement matrix is obtained as the new grayscale similarity weight of the bilateral filtering, and the spatial neighboring weights remain unchanged. The algorithm flow is shown in Figure 2.

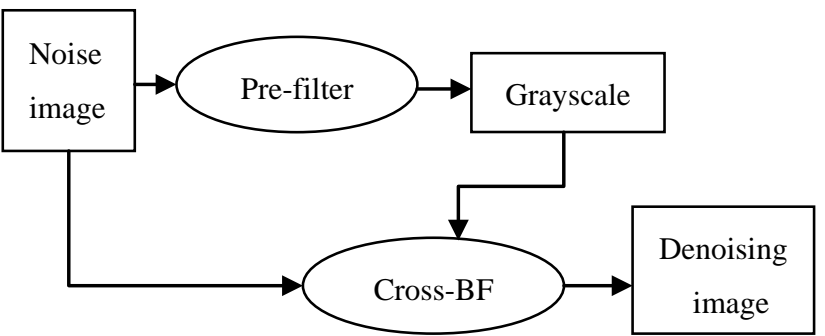

Figure 2. Block diagram of the cross-bilateral filter In this paper, the stationary wavelet transform (SWT) algorithm was used as the pre-filter. Some parameters in the SWT algorithm were set as follows: the wavelet basis function selected "symlet" wavelet which was approximately symmetric, and the decomposition scale was 3. According to the characteristics of wavelet transform and noise normal distribution of road image, the threshold value in this paper was set to $\mathrm{k}=3 \sigma_{n}$, wherein the noise standard deviation $\sigma_{n}$ $\left(\sigma_{n}{ }^{2}=\operatorname{median}(|W|) / 0.6745\right)$ was estimated by the first layer high frequency coefficient after wavelet decomposition. Soft threshold method was adopted for threshold processing. Wavelet threshold denoising belongs to frequency domain denoising method, bilateral filtering belongs to spatial domain denoising method, and the cross-bilateral filtering algorithm combines them to achieve better denoising effect.

\subsection{A Simplified PCNN Model Based on Markov Network Threshold (SPCNN)}

\subsubsection{PCNN Model and Its Basic Principle}

Eckhorn(1990) proposed a neural network model based on neuronal signal transmission characteristics by observing the phenomenon of synchronous pulse emission in the cerebral cortex of cats, and thus developed the pulse-coupled neural network (PCNN) model. When PCNN is applied to image processing, the pixels of two-dimensional input image correspond to the network neurons one by one, and the ignition neurons will stimulate the adjacent neurons by coupling with 
the neighboring neurons, and if the internal activity of the adjacent neuron is greater than or equal to the threshold, it will be captured and ignited. That is, when the gray value of the current pixel is close to that of the neighborhood pixel, it will cause the ignition of the neighborhood pixel, which is called the capture characteristic and the synchronization pulse release characteristic of PCNN network, and the image segmentation can be achieved by using this characteristic. The mathematical model can be expressed as:

$$
\left\{\begin{array}{c}
F_{i j}(n)=e^{-\alpha_{F}} F_{i j}(n-1)+V_{F} \sum_{k, l} M_{i j k l} F_{k l}(n-1)+S_{i j} \\
L_{\mathrm{ij}}(n)=e^{-\alpha_{L}} L_{i j}(n-1)+V_{L} \sum_{k, l} W_{i j k l} Y_{k l}(n-1) \\
U_{i j}(n)=F_{i j}(n)\left(1+\beta L_{\mathrm{ij}}(n)\right) \\
\theta_{i j}(n)=e^{-\alpha_{\theta}} \theta_{i j}(n-1)+V_{\theta} Y_{i j}(n-1) \\
Y_{i j}(n)= \begin{cases}1 & U_{i j}(n) \geq \theta_{i j}(n) \\
0 & U_{i j}(n)<\theta_{i j}(n)\end{cases}
\end{array}\right.
$$

Where $\beta$ and $n=$ Coefficient of link and number of iterations $i j$ and $k l=$ any point and its adjacent points in the image

$F_{i j}, L_{i j}, U_{i j}$ and $\theta_{i j}=$ feedback input, link input, internal activity item and dynamic threshold respectively

$V_{F}, V_{L}$ and $V_{\theta}=$ connection weight amplification coefficient of the corresponding subsystems

$\alpha_{F}, \alpha_{L}$ and $\alpha_{\theta}=$ iterative decay time constant of the corresponding subsystems

$\mathrm{M}$ and $\mathrm{W}=$ the link weight matrix of the center point pixel and the neighborhood pixel

$S_{i j}$ and $Y_{i j}(n)=$ gray value of a pixel and output

\subsubsection{SPCNN Algorithm}

\section{(1) Simplified PCNN Model (SPCNN)}

The traditional PCNN model has many parameters, the number of iterations is difficult to determine, and the segmentation result is closely related to parameter selection, so it is difficult to achieve the adaptive segmentation of PCNN.

$$
\left\{\begin{array}{c}
F_{i j}(n)=S_{i j} ; \theta_{i j}(n)=\theta_{m} \\
L_{\mathrm{Eij}}(n)=\sum_{k, l} M_{i j k l} Y_{k l}(n-1) \\
L_{\mathrm{Iij}}(n)=\sum_{k, l} M_{i j k l} \overline{Y_{k l}(n-1)} \\
L_{\mathrm{Sij}}(n)=L_{\mathrm{Eij}}(n)+L_{\mathrm{Iij}}(n) \\
U_{i j}(n)=F_{i j}(n)\left(1+\beta L_{\mathrm{ij}}(n)\right) \\
Y_{i j}(n)= \begin{cases}1 & U_{i j}(n) \geq \theta_{i j}(n) \\
0 & U_{i j}(n)<\theta_{i j}(n)\end{cases}
\end{array}\right.
$$

Where $L_{\mathrm{Sij}}(n)=$ a combination of neuronal excitatory link input $L_{\mathrm{Eij}}(n)$ and neighborhood inhibitory link input $L_{\mathrm{Iij}}(n)$ In order to realize the adaptive and real-time performance of the segmentation algorithm, this paper simplifies and improves the model (SPCNN) is shown in equation (3).

The exponential decay mechanism of the PCNN threshold function is independent of grayscale, which brings certain difficulties to image segmentation with low contrast $(\mathrm{Xu}$ et al.,2011), so the exponential decay mechanism is removed after simplification. The link input $L_{\mathrm{Sij}}(n)$ can improve the information capacity acquired by neurons and improve the image segmentation effect. SPCNN model only needs to determine three parameters $\mathrm{M}, \beta$ and $\theta_{m}$, which will described in detail in the next section.

(2) Three Main Parameters

1) M takes the inverse of square of the distance between pixels.

2) The tightness of the connection between $F$ and $L$ channels of PCNN neurons is regulated by $\beta$. The larger the $\beta$ value, the larger the range of synchronous ignition. However, the same $\beta$ is not conducive to the preservation of image detail information, so in order to improve the segmentation effect, $\beta$ can be represented by a feature related to the image gradation. Since the energy of Laplace (EOL) can effectively reflect the edge details, texture changes and other information of the image area, this paper uses EOL of each pixel to replace $\beta$ to achieve the adaptive setting of $\beta$. EOL of pixel $(i, j)$ is defined as follows:

$$
E O L=\sum_{(X, Y) \in w}\left(f_{x x}+f_{y y}\right)^{2}
$$

Where

$$
\begin{aligned}
& f_{x x}+f_{y y}=f(x+1, y)+f(x-1, y)+f(x, y+ \\
& 1)+f(x, y-1)+4 f(x, y) \\
& f(x, y)=\text { gray value at point }(\mathrm{x}, \mathrm{y}) \\
& \mathrm{w}=\text { a window range with size of } 3 * 3
\end{aligned}
$$

3) The Markov network threshold $\theta_{m}$. The Decomposable Markov Network (DMN) is a probabilistic network that does not focus on the inference relationship between individual nodes of the network, but emphasizes the group structure behavior of individual nodes and related nodes, and uses this group structure behavior to determine the state output of a single node. The state output, which makes it possible to reveal the statistical characteristics of the pixel grayscale and its spatial relationship within the image. The image is characterized by a single pixel being strongly influenced by its neighboring pixels while maintaining its independence. The state of each pixel is never simply related to itself or several 
neighboring pixels, but a set of states with significant statistical and random properties(Cao,2005), and DMN is a suited representation of this set. The threshold signal $\theta_{k}(t)$ generator can be expressed as:

$$
\theta_{k}(t)=\left\{\begin{array}{c}
V_{k} \exp \left(-\frac{t-t_{1}}{\tau_{k}}\right), \quad t_{1}<t<t_{2} \\
V_{k}, t=t_{1} 、 t_{2}
\end{array}\right.
$$

Where $\quad V_{k}=$ a predetermined increment value

$\tau_{k}=$ the time decay constant

$\mathrm{t}=$ the time parameter

When a PCNN neuron fires, its $\theta_{k}(t)$ generator is assigned a value of $V_{k}$, which is the higher the better, so that the threshold signal value in front of the neuron can be ignored to act as a blocking effect. It can be assumed that for the internal behavior of the neuron, the value of $V_{k}$ is greater than any possible value. If the time at which the two neurons successively generate pulses is $t_{1}$ and $t_{2}$, then equation (5) exactly expresses the operation of the threshold signal generator.

The $\theta_{k}(t)$ in the above equation was directly replaced by gray value as input signal $x$ (Zhang et al.,2004), derived from this:

$$
\mathrm{T}(\mathrm{t})=\tau_{k} \ln \left(V_{k} / x\right), \mathrm{t}_{1}<t<t_{2}
$$

Where $\tau_{k}=\frac{1}{x}$, a function of the gradation value

$$
V_{k}=X_{\text {sum }} \text {, the sum of all gradations in the image }
$$

Equation (6) can also be regarded as a composite representation of two systems, which are equivalent to the static system $\mathrm{T}_{1}(\mathrm{t})=\tau_{k} \ln \left(V_{k}\right)$ aland the dynamic system $\mathrm{T}_{2}(\mathrm{t})=\tau_{k} \ln x$ superposition. After the equivalent conversion, can be obtained:

$$
\mathrm{T}(\mathrm{t})=\frac{1}{x} \ln \left(\frac{1}{x} / \frac{1}{X_{\text {sum }}}\right)\left(\mathrm{t}_{1}<t<t_{2}\right)
$$

It can be found that equation (7) is actually the cross entropy composed of the image static system $\left(\mathrm{P}_{\mathrm{X}}=1 / X_{\text {sum }}\right)$ and the dynamic system $\left(\mathrm{P}_{x}=1 / x\right)$. On the one hand, the static system probability distribution attempts to homogenize the image gray scale; on the other hand, the dynamic system probability distribution tries to preserve the change information of the original image gray level as much as possible. Image segmentation can be performed by the process of interaction between them to find local grayscale inconsistencies. The pixel gray value is regarded as the maximum entropy value of the corresponding DMN node and each network node is excited at the same time. In this way, all values of the node variable space are excited to maximum state, and the average deviation value $\theta_{m}$ between the maximum entropy value (grayscale value) of all nodes and their initial values at this time is calculated as the threshold for network neighbor connection search. $\theta_{m}$ (Markov network threshold) proposed in this paper can be expressed as:

$\theta^{\text {matrix }}=x-\frac{1}{x} \ln \left(\frac{X_{\text {sum }}}{x}\right) ; \theta_{m}=\operatorname{mean}\left(\left|\theta^{\text {matrix }}\right|>0\right)$

\section{(3) SPCNN Algorithm Steps}

The specific steps of crack image segmentation as follows:

1) Input: link field $F$ is the image pixel value, initializing the following values: $F_{i j}(0)=L_{i j}(0)=Y_{i j}(0)=U_{i j}(0)=\theta_{i j}(0)$; 2) The EOL of each pixel is calculated according to equation (6) as the link strength value $\beta$ of the corresponding neuron;

3) The Markov network grayscale threshold value $\theta_{m}$ is calculated according to the equation (8) as the boundary value between the crack and pavement background;

4) Calculates equations (6) (8) successively, and obtains the segmentation result of current pavement crack image $(\mathrm{Y})$.

\section{EXPERIMENT RESULTS AND ANALYSIS}

\subsection{Filter Experimental Results and Analysis}

\subsubsection{Objective Evaluation}

Commonly used evaluation indicators are Mean Square Error (MSE) and Peak Signal-to-noise Ratio (PSNR). They are defined by the following equation:

$$
\left\{\begin{array}{c}
\text { MSE }=\frac{1}{M N} \sum_{i=1}^{M} \sum_{j=1}^{N}[o(i, j)-f(i, j)]^{2} \\
\text { PSNR }=10 \log _{10}\left[\left(\frac{1}{M N} \sum_{i=1}^{M} \sum_{j=1}^{N} L^{2}\right) / M S E\right]
\end{array}\right.
$$

Where $o(i, j)=$ standard images; $f(i, j)=$ filtered images $\mathrm{M}, \mathrm{N}=$ the image size; $\mathrm{L}=$ the gray range of image 
It is difficult to evaluate the quality of crack image after denoising because of the corresponding standard image cannot be obtained. In order to objectively evaluate denoising performance of cross-bilateral filtering algorithm, white noise with a standard deviation of 20 was artificially added with Lena image that commonly used in the field of image processing, and then original $\mathrm{BF}$ and cross-BF method filtered it respectively. Experimental result was shown in Figure 3:

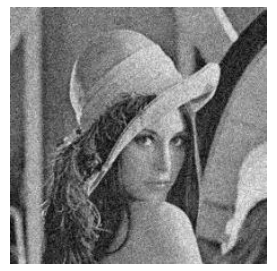

(c1) original image

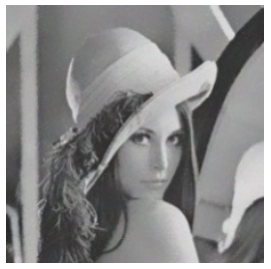

(c3) original $\mathrm{BF}$

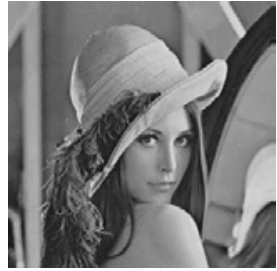

(c2) Add white noise

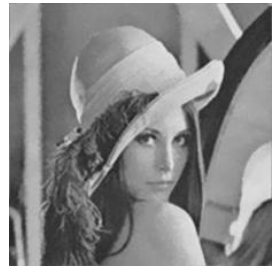

(c4) Cross-BF

Figure 3. Smoothing results of BF and Cross-BF

In this paper, PSNR was used as the filtered image quality evaluation index, the values as shown in figure 4:

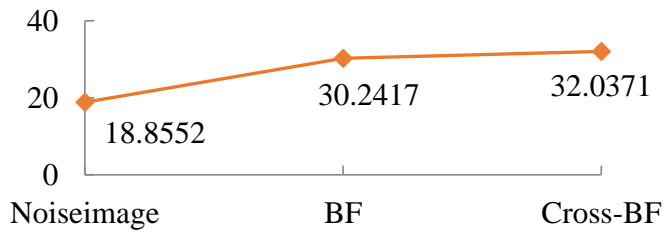

Figure 4. PSNR values of two filtering algorithms

As could be seen from Figure 4, PSNR of the image with added noise was 18.8552, PSNR of the filtered image was greatly improved by the two bilateral filtering methods, and detail of the image was well maintained. Cross-BF algorithm had the highest PSNR value of 32.0371, which was better than the original $\mathrm{BF}$ algorithm.

\subsubsection{Subjective Evaluation}

The crack image was filtered by the three methods in this paper, and the result was shown in Figure 5:

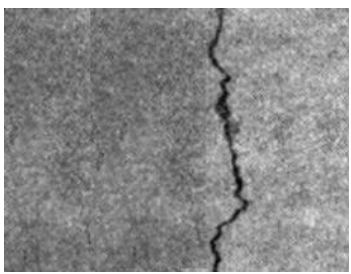

(d1) Original image

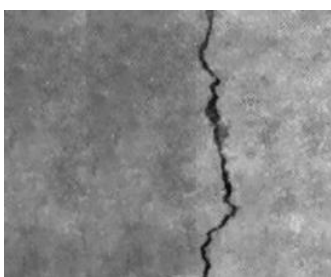

(d2) BF

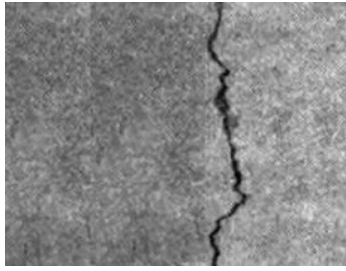

(d3) SWT

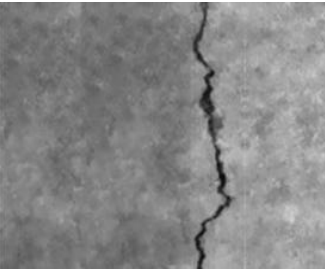

(d4) Cross-BF
Figure 5. Filtered results by BF, SWT and Cross-BF

From the above image results, it could be found that after BF and Cross-BF filtering, the image was smoothed while well preserving the details of the edge of the crack target, and the effect of Cross-BF was better that BF algorithm. SWT smoothing effect was slightly worse, indicated that cross-BF algorithm had a good effect of denoising while protecting the edge for bridge crack images.

\subsection{Segmentation Experiment Results and Analysis}

Two typical bridge crack images (test1 and test2) were selected as test images to verify the effectiveness of the proposed method in MATLAB R2014a environment, all images use 3*3 neighborhood link weight matrix: $M=\left[\begin{array}{ccc}0.5 & 1 & 0.5 \\ 1 & 0 & 1 \\ 0.5 & 1 & 0.5\end{array}\right]$. By comparing the segmentation results of the classical global threshold segmentation algorithm OTSU, the original PCNN segmentation algorithm and the SPCNN algorithm in this paper, the advantages and disadvantages of SPCNN algorithm were evaluated from three aspects: visual effect, segmentation performance and operation time. The segmentation results are shown in Figure 6:

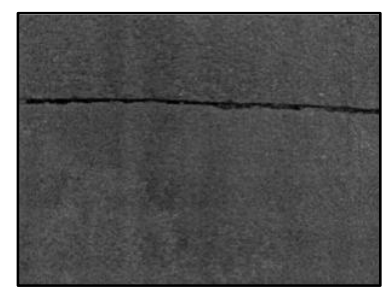

(e1) test1 $(400 * 300)$

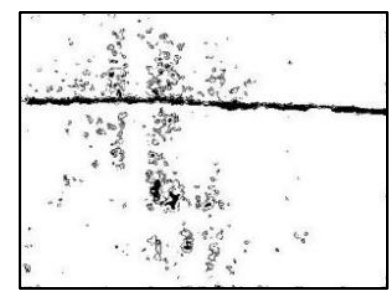

(e3) PCNN

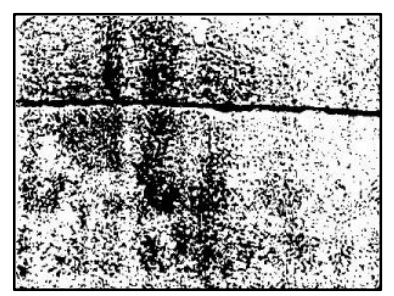

(e2) OTSU

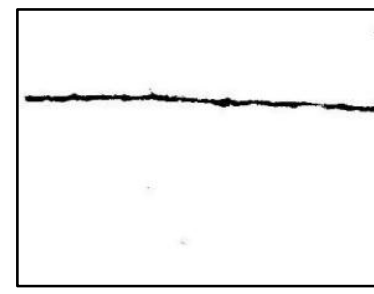

(e4) SPCNN 


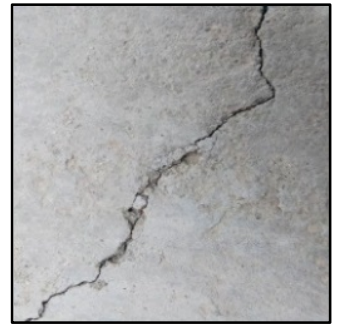

(f1) test2(1024*1024)

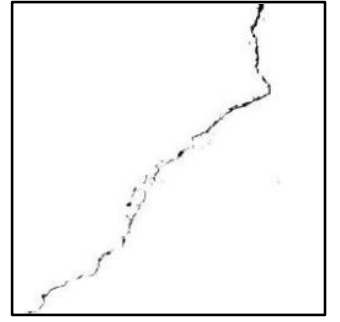

(f3) PCNN

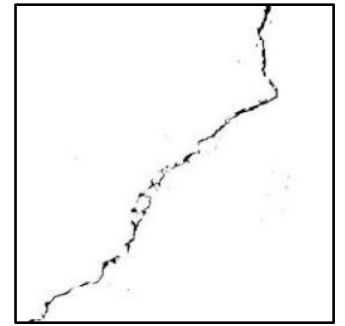

(f2) OTSU

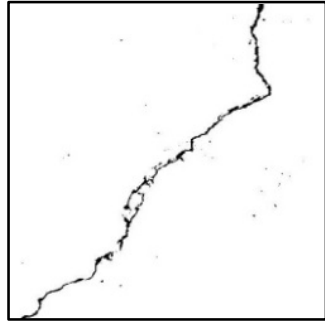

(f4) SPCNN
Figure 6. Segmentation results of uneven illumination images by three segmentation methods

\subsubsection{Subjective Visual Effects Comparison}

Compared the segmentation results of the two uneven illumination bridge pavement images, in terms of subjective vision, OTSU was greatly affected by environmental factors and the segmentation effect was unstable. The optimal iteration times of PCNN segmentation was difficult to determine. It spent a lot of time, and there were still many false extracts and faults in the segmentation results. Compared with the previous two segmentation methods, the SPCNN model algorithm proposed in this paper had the best segmentation effect. Although the crack target extracted in figure 4(f4) had slight fracture, the overall segmentation result was good.

\subsubsection{Objective Segmentation Performance Comparison}

In this paper, results of manual identification were taken as the standard to evaluate the performance of the above three algorithms by calculating the correct extraction rate $P_{t}$ and error rate $P_{f}$. The larger the value of $P_{t}$ or the smaller the $P_{f}$, the better the effect.

$$
P_{t}=T N / N_{V P} ; P_{f}=F N / N_{u v p}
$$

Where $\mathrm{TN}=$ the number of pixels correctly segmented for the crack target

$\mathrm{FN}=$ the number of pixels mistakenly divided into cracks in the image of experimental results
$N_{V P}$ and $N_{u v p}=$ the number of crack pixels and non-crack pixels in the standard crack segmentation image The above three methods were used for segmentation of 100 images captured, and the accuracy and error rate of segmentation results were averaged. It could be found that SPCNN had higher $P_{t}$ (up to $91 \%$ ) and lower $P_{f}$ (down to $10 \%$ ). As shown in Figure 7:

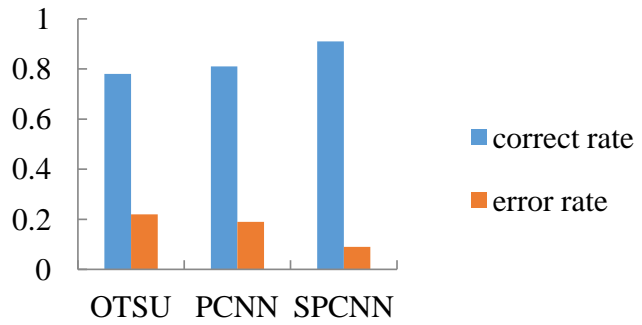

Figure 7. Accuracy and error rate of three segmentation algorithms

(3) Computational Time Comparison

Repeated the experiment 15 times on the image test1, and took the average of the operation time of the three algorithms, as shown in Figure 8. It could be concluded that the algorithm spent less time than the original PCNN and is equivalent to the OTSU. SPCNN could reduce the computational time on the basis of ensuring the segmentation effect.

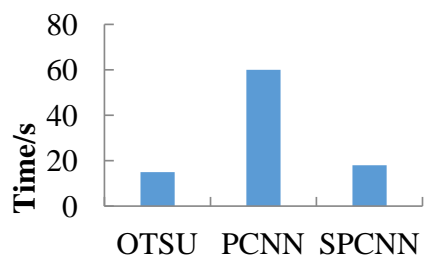

Figure 8. Time consumption of three segmentation algorithms

\section{CONCLUSIONS}

For the uneven illumination bridge pavement crack images, this paper first analyzed the principle of image illumination, used wavelet transform to remove the influence of uneven illumination, and realized image enhancement. Next SWT was used as pre-filtering processor to improve bilateral filtering. Finally, in the processing of image segmentation, combined with the synapse integration characteristics and image gray characteristics, the traditional PCNN were improved, PCNN segmentation method based on the Markov network threshold (SPCNN) was proposed. Experimental results showed that the proposed algorithm had obvious advantages in subjective visual 
effects, objective segmentation performance and time consuming. It validates the effectiveness of the proposed algorithm on image segmentation of uneven illumination bridges pavement cracks. From the experimental results of this paper, the following three conclusions were drawn:

(1) Compared with the histogram equalization, the enhancement algorithm of this paper removed the influence of illumination well and had advantages for the subsequent segmentation processing.

(2) The cross-bilateral filtering algorithm could improve the image signal-to-noise ratio from 18.855 to 32.037 , which was better than the original bilateral filtering algorithm.

(3) The average segmentation accuracy rate of segmentation of SPCNN algorithm was more that $90 \%$. Compared with the traditional PCNN method, this method is better in subjective visual effects and objective segmentation performance, less time consuming.

\section{REFERENCES}

Cao, J. N., 2005. Study on approach of image segmentation based on decomposable markov networks. Wuhan University.

Chambon, S., Moliard, J., 2011. Automatic road pavement assessment with image processing: review and comparison. International Journal of Geophysics, Article ID989354.

Eckhorn, R., 1990. Feature linking via synchronization among distributed assemblies: simulations of results from cat visual cortex. Neural Computation, 2(3): 293-307.

He, Y. Q., Qiu, H. X., 2012. A method of cracks image segmentation based on the means of multiple thresholds. Journal of Communication and Computer, 9(10): $1147-1151$.

Liu, F. F., Xu, G. A., Yang, Y. X., Niu, X. X., Pan, Y. L., 2008. Novel approach to pavement cracking automatic detection based on segment extending. International Symposium on Knowledge Acquisition and Modeling.

Li, Q. Q., Zou, Q., Mao, Q. Z., 2010. Pavement crack detection based on minimum cost path searching. China Journal of Highway and Transport, 23(06): 28-33.
Lee, B. Y., Kim, Y. Y., Yi, S. T., Kin, J. K., 2013. Automated image processing technique for detecting and analysing concrete surface cracks. Structure and Infrastructure Engineering, 9(6): 567-577.

Nishikawa, T., Yoshida, J.,.Sugiyama, T. Fujino, Y., 2012. Concrete crack detection by multiple sequential image filtering. Computer-Aided Civil and Infrastructure Engineering, 27(1): 29-47.

Oliveira, H., Correia., P. L., 2013. Automatic road crack detection and characterization. IEEE Transactions on Intelligent Transportation Systems, 14(1): 155-168.

Wu, J. P., Yang, Z. X., Han, D., Bai, Z. F., Su, Y. T., 2010. 2D barcode image binarization based on wavelet and otsu method. Computer Engineering, 36(10):190-192.

Wang, D. F., Zeng, W. M., Wang, N. Z., Road crack detection under uneven illumination using improved k-means algorithm. Computer Applications and Software, 32(7): 244-247.

Xu, J. J., Gao, S., Bi, D. Y., Chen, Y., 2011. Novel image segmentation algorithm. Journal Of Xidian University, (1):8-15.

Yusuke, F., Yoshihiro, M., Yoshihiko, H., 2006. A method for crack detection on a concrete structure. The 18th International Conference on Pattern Recognition, 901-904.

Zhang, J. Y., Lu, Z. J., Shi, L., Dong, J. Y., 2004. Salt and pepper noise image filtering based on pulse-coupled neural network. SCIENCE IN CHINA Ser. E Information Sciences, 34(8):882 894

Zhang, D. J., Li, Q. Q., Chen, Y., Cao, M., He, L., 2016. Asphalt pavement crack detection based on spatial clustering feature. ACTA AUTOMATICA SINICA, 42(3).

Zhao, S. S., He, N., 2017. Road surface crack detection based on CNN. Transducer and Microsystem Technologies, (11): 140-143. 